\title{
EFFECTS OF ROTATION ON THE MINIMUM MASS OF PRIMORDIAL PROGENITORS OF PAIR-INSTABILITY SUPERNOVAE
}

\author{
E. Chatzopoulos and J. Craig Wheeler \\ Department of Astronomy, University of Texas at Austin, Austin, TX, USA; manolis@astro.as.utexas.edu \\ Received 2011 December 12; accepted 2012 January 6; published 2012 March 5
}

\begin{abstract}
The issue of which stars may reach the conditions of electron/positron pair-formation instability is of importance to understand the final evolution both of the first stars and of contemporary stars. The criterion to enter the pair-instability regime in density and temperature is basically controlled by the mass of the oxygen core. The main-sequence masses that produce a given oxygen core mass are, in turn, dependent on metallicity, mass loss, and convective and rotationally induced mixing. We examine the evolution of massive stars to determine the minimum main-sequence mass that can encounter pair-instability effects, either a pulsational pair-instability supernova (PPISN) or a full-fledged pair-instability supernova (PISN). We concentrate on zero-metallicity stars with no mass-loss subject to the Schwarzschild criterion for convective instability, but also explore solar metallicity and mass loss and the Ledoux criterion. As expected, for sufficiently strong rotationally induced mixing, the minimum main-sequence mass is encountered for conditions that induce effectively homogeneous evolution such that the original mass is converted almost entirely to helium and then to oxygen. For this case, we find that the minimum main-sequence mass is about $40 M_{\odot}$ to encounter PPISN and about $65 M_{\odot}$ to encounter a PISN. The implications of these results for the first stars and for contemporary supernovae are discussed.
\end{abstract}

Key words: stars: evolution - stars: individual (pair instability supernovae) - stars: mass-loss - supernovae: general

Online-only material: color figures

\section{INTRODUCTION}

Very massive stars were long ago predicted to get hot enough that the ambient photons in the interior are sufficiently energetic to create electron/positron pairs (Rakavy \& Shaviv 1967, 1968; Barkat et al. 1967; Rakavy et al. 1967; Fraley 1968; see also Wheeler 1977; El Eid \& Hilf 1977; El Eid et al. 1983; Ober et al. 1983; Bond et al. 1984; Carr et al. 1984; Stringfellow \& Woosley 1988). The conversion of energy to rest mass rather than thermal energy alters the equation of state (EOS) so that the pressure does not increase sufficiently with density upon compression to maintain hydrostatic equilibrium. If a sufficiently large, massaveraged region of the star is in the pair-formation regime, such that $\left\langle\Gamma_{1}\right\rangle<4 / 3$, the structure will be dynamically unstable. Models predict that this happens after stars have undergone their central helium burning and have formed massive cores composed primarily of oxygen (with a small mass fraction of carbon). In models, the instability occurs in an off-center shell. The rapid contraction of this shell drives it inward, leading to the rapid compression and heating of the inner core of oxygen. Unlike the case of iron-core collapse (CC), the oxygen in these stars is subject to strong energy release by rapid thermonuclear burning. The result is the prediction that the star is totally disrupted, leaving no remnant, but with the production of a very large mass of radioactive ${ }^{56} \mathrm{Ni}$, the decay of which could power the light output.

The original calculations, cited above, found that the pairinstability regime was encountered for stars with massive oxygen cores, greater than about $60 M_{\odot}$. At the time, it was not clear how massive a star needed to be to develop a sufficiently massive oxygen core, but estimates were in the range of $100 M_{\odot}$. It was also not clear that any stars sufficiently massive to reach this condition of instability existed. The latter point was resolved theoretically with the understanding that massive, radiation-pressure-dominated stars subject to dynamical insta- bility would be stabilized in the nonlinear regime (Appenzeller 1970; Ziebarth 1970) and observationally with the discovery that young clusters such as R 136 in 30 Doradus contained very massive stars (Panagia et al. 1983; Hunter et al. 1995; Crowther et al. 2010). A subsequent important development was the prediction that in the context of $\Lambda \mathrm{CDM}$ models of the universe, the first stars forming at zero metallicity after the Dark Ages might preferentially form especially massive stars that would, in turn, be subject to pair instability (Abel et al. 1998, 2000; Bromm et al. 2002; Bromm \& Larson 2004).

Considerable effort has gone into the computation of the formation of the first stars, the evolution of stars that will reach pair-instability conditions, and the predicted observational properties of the resulting explosions (Heger \& Woosley 2002, 2010; Ohkubo et al. 2003, 2009; Scannapieco et al. 2005; Woosley et al. 2007; Waldman 2008; Kasen et al. 2011; Whalen \& Fryer 2010; Joggerst \& Whalen 2011). Pair-instability supernovae (PISNe) models have a characteristic nucleosynthetic yield (Heger \& Woosley 2002; Ohkubo et al. 2003), but searches for evidence of such a distribution in the lowest metallicity stars have not revealed the expected pattern (Christlieb et al. 2002; Frebel et al. 2005). In general, lower metallicity will suppress mass loss and allow relatively lower mass mainsequence stars to encounter the pair-instability regime. For a given main-sequence mass, higher metallicity will tend to lead to lower mass oxygen cores, thus avoiding this regime. Two subsequent developments have altered the context in which pair instability is considered. One is the recent understanding that conditions of the first stars may be more susceptible to fragmentation so that the first stars may have been of smaller mass than once thought (Stacy et al. 2010; Greif et al. 2011). Another important development was the discovery in the contemporary universe of a category of super-luminous supernovae (SLSNe) that are relatively rare, but brighter by a factor of 10-100 than most contemporary supernovae ( $\mathrm{SNe}$; 
Smith et al. 2007; Quimby et al. 2007; Miller et al. 2009; Gal-Yam et al. 2009; Chatzopoulos et al. 2011). Some of these SLSN show evidence of high mass, but cannot be PISNe (Smith et al. 2007; Miller et al. 2009; Chatzopoulos et al. 2011, 2012; Leloudas et al. 2012). If their brightness derived from the radioactive decay of ${ }^{56} \mathrm{Ni}$, as demanded by the pair-instability model, they would require a greater mass of ${ }^{56} \mathrm{Ni}$ than is allowed for the total mass of the ejecta. The ejecta mass is constrained by the width of the light curve (LC) that is a measure of the diffusion time. The great luminosity of SLSNe like SN 2006gy probably derives from the collision of the ejecta with a shell of matter previously ejected by the progenitor star (Smith et al. 2007; Chevalier \& Irwin 2011; Chatzopoulos et al. 2011). Several of these super-luminous events do, however, have all the characteristics expected of a PISN (SN 2007bi, Gal-Yam et al. 2009; PTF 10nmn, O. Yaron et al. in preparation). These events have occurred in low, but not zero, metallicity environments. The possibility that the first stars are not of especially high mass and the discovery of contemporary SLSNe that have the characteristics of PISN brings a new focus to the issue of just which stars can undergo pair instability and under what conditions.

Maeder (1987; see also Maeder \& Meynet 2011 for a review) discussed the effects of rotationally induced mixing on the evolution of massive stars. He concluded that there would be substantial mixing produced by the small-scale three-dimensional tail of the turbulent spectrum of the baroclinic instability and that the diffusion coefficient could be sufficiently large to mix most massive stars during their main-sequence lifetimes. In particular, above a critical rotation, Maeder predicted that the evolutionary tracks go upward and blueward, very close to those of fully homogeneous evolution. The immediate implication was that stars in the mass range predicted to reach pair instability (already radiation-pressure dominated and hence close to neutral dynamical instability) were susceptible to quasi-homogeneous evolution such that nearly all the main-sequence mass could be burned to heavier elements. Quasi-homogeneous evolution would significantly decrease the main-sequence mass that leads to pair instability. The notion that quasi-homogeneous evolution might lead to larger core masses has been applied in the context of the progenitors of gamma-ray bursts (GRBs; Heger et al. 2005; Yoon \& Langer 2005) and explored to a certain extent in recent work (Heger et al. 2000; Langer et al. 2007; Ekström et al. 2008, 2011; Brott et al. 2011a, 2011b), but has not been pursued in detail in the context of pair instability.

In this paper, we conduct a thorough parameter study to explore the minimum main-sequence mass that encounters the pair-instability regime. In his unpublished $\mathrm{PhD}$ thesis, Barkat et al. (1967) noted that some models that skirted the pairinstability regime ejected shells of matter, but survived the instability and continued to evolve. Somewhat higher mass models became sufficiently unstable that a dynamical explosion, a PISN, was produced directly. Woosley et al. (2007) invoked the former effect, which they called a pulsational pair-instability supernova (PPISN) to address the nature of SLSN 2006gy. Their simulations showed a repeated ejection of shells. The second, faster shell collided with the first and produced a luminous display reminiscent of the LC of SLSN 2006gy. Here we will delineate models that encounter the PPISN and those that encounter the full PISN explosion. Section 2 describes our assumptions and models, Section 3 gives the results and presents a model that invokes a different mixing criterion and another with solar metallicity and mass loss. Section 4 discusses our conclusions.

\section{MODELS}

As discussed in the Introduction, the masses of stars that evolve from the main sequence to either PPISN or PISN will be a function of metallicity, mass loss, and rotationally induced mixing. The outcome will also depend on the treatment of convective instability, semi-convection, and overshoot. Dissipation of shear by magnetic effects may also attend the rotationally induced mixing. Rather than explore this whole parameter space where the physics is, in any case, uncertain, we have focused on the portion of parameter space that is expected to lead to the minimum mass to encounter pair formation. In particular, we have explored conditions of zero metallicity and have neglected mass loss for the majority of our models in order to establish the proof of principle. We have run a few models at solar metallicity in order to put our results in context. We have adopted rates of rotation on the main sequence that run from non-rotating to rotating at $80 \%$ of equatorial Keplerian velocity. We have focused on Schwarzschild convection for two reasons. One is that this prescription will tend to enhance the mass of the oxygen core for a given main-sequence mass, in keeping with the philosophy of this exploratory work. The second reason is based on a suspicion that neither the Schwarzschild nor the Ledoux criterion really captures the three-dimensional, plume-driven convection in real stars. As we will describe in a future work (E. Chatzopoulos et al. in preparation), we have grounds to believe that in the late stages, when the oxygen core forms, the Schwarzschild criterion is the more appropriate. Multi-dimensional effects tend to swamp the stabilizing effect of composition gradients. Our simulations have also adopted the effects of magnetic viscosity as parameterized by Heger et al. (2005) based on the prescriptions of Spruit $(1999,2002)$. This is not because we believe that this particular parameterization captures all the relevant multi-dimensional MHD instabilities and related phenomena in rotating, shearing, stars, but because it is a widely used and recognized algorithm so that our results can be readily compared to others using the same prescription.

We have used the Modules for Stellar Experiments in Astrophysics (MESA; Paxton et al. 2011) code to calculate the evolution of a grid of $Z=0$ massive stars ranging from zeroage main sequence (ZAMS) mass of $35 M_{\odot}$ to $200 M_{\odot}$ for four different degrees of ZAMS rotation. We assume initially rigid body rotation on the ZAMS with surface rotation corresponding to $0 \%, 30 \%, 50 \%$, and $80 \%$ of the critical Keplerian rotation $\Omega_{\text {crit }}=(g(1-\Gamma) / R)^{1 / 2}$, where $g=G M / R^{2}$ is the gravitational acceleration at the "surface" of the star, $G$ the gravitational constant, $M$ the mass, $R$ the radius of the star, and $\Gamma=L / L_{\mathrm{Ed}}$ the Eddington factor where $L$ and $L_{\mathrm{Ed}}$ are the total radiated luminosity and the Eddington luminosity, respectively. The $35 M_{\odot}$ model was only run for maximum rotation $(80 \%)$ in order to establish the lower mass limit for PPISN and the models above $85 M_{\odot}$ for lower $(30 \%)$ and zero degrees of rotation to establish the corresponding minimum ZAMS mass for PPISN. The models with ZAMS masses $40-85 M_{\odot}$ were run in bins of $5 M_{\odot}$ for all the selected degrees of rotation in order to better resolve the limits for different final fates of the stars. In order to benchmark against the results of Heger \& Woosley (2002) and Woosley et al. (2007) for non-rotating stars that will produce a pure PISN and a PPISN, respectively, we have run a $110 M_{\odot}$ and a $200 M_{\odot}$ model (ZAMS masses) without rotation. The reason that we chose this range of ZAMS masses is the fact that Heger \& Woosley (2002) and Heger et al. (2003) predict that oxygen core masses $\left(M_{\mathrm{O}-\text { core }}\right)$ ranging from 40 to $64 M_{\odot}$ will undergo PPISN while $64<M_{\mathrm{O}-\text { core }}<133 M_{\odot}$ will explode as direct PISNe for 
non-rotating progenitors, but with significantly rapid rotation a lower ZAMS mass can produce oxygen core masses in this range.

MESA was run with the Schwarzschild criterion for convection implemented for reasons discussed above and for zero mass loss. It should be mentioned that rotationally induced mass loss may reduce the final oxygen core masses. Ekström et al. (2008) found that rapidly rotating zero-metallicity stars with ZAMS masses above $\sim 50 M_{\odot}$ may lose up to $\sim 11 M_{\odot}$; however, due to the lack of understanding of mass-loss mechanisms in massive stars, this remains uncertain. Nevertheless, we also conducted calculations with mass loss included in models with initially zero metallicity, as described in Section 3.2, to estimate the impact of mass loss on the final fate of the models. MESA employs a combination of prescriptions for the EOS, but for high density and temperature plasma the HELM EOS (Timmes \& Swesty 2000) is used. The HELM EOS accounts for pressure induced by radiation, ions, electrons, positrons, and corrections for Coulomb effects and therefore for the effects of electron-positron pair formation, which drives the adiabatic in$\operatorname{dex} \Gamma_{1}$ below 4/3. For the treatment of nuclear processes with MESA we employ the "approx21" network (Timmes 1999), which covers all major stellar nuclear reaction rates. The effects of angular momentum transport via rotation and magnetic fields are treated based on the one-dimensional (1D) approximations of Spruit $(1999,2002)$ and Heger et al. (2005).

MESA is capable of running stellar models up to the CC and pre-supernova (pre-SN) stage; however, for high-mass stellar models that encounter a degree of instability induced by electron-positron pair production the effects become very dynamic and a challenge for a stellar evolution code to handle. Future expansions of MESA will be able to handle those dynamical effects and to follow the SN explosions of stars with 1D hydrodynamics implemented (B. Paxton 2011, private communication). In the current work, stellar models for which a significant fraction of their core approaches the $\Gamma_{1}<4 / 3$ regime due to pair formation are stopped before core oxygen ignition and within the carbon-burning phase, shortly before becoming dynamic. Those models are then mapped into the multi-dimensional hydrodynamics code FLASH (Fryxell et al. 2000), and their evolution is followed in 1D. The newest version of FLASH (FLASH4-alpha release) is used for these simulations. FLASH is very suitable to follow the dynamical transition of the models from MESA because it uses the same EOS (HELM; Timmes \& Swesty 2000) and similar nuclear reaction network. PPISNe are characterized by a violent contraction and pulsation that heats the core up to a temperature such that some of the oxygen is burned to produce primarily ${ }^{28} \mathrm{Si}$ and ${ }^{32} \mathrm{~S}$ (Woosley et al. 2007). Pure thermonuclear PISNe are heated significantly enough from the dynamical collapse induced by the softening of the EOS due to electron-positron pair formation that they burn oxygen explosively and large amounts of ${ }^{56} \mathrm{Ni}$ are produced. FLASH is capable of reproducing those basic features of the events and is therefore used to establish the final fate of the models. We note that in the 1D FLASH hydrodynamic simulations the effects of rotation are not considered. In a future work we plan to investigate the effects of rotation in the hydrodynamical stage of PISNe with multi-dimensional FLASH simulations.

\section{RESULTS}

Table 1 summarizes the characteristics of all the models considered in this work (for zero metallicity and mass-loss turned off). The first column lists the ZAMS mass ( $M_{\mathrm{ZAMS}}$, in solar masses), the second is the critical rotational ratio $\Omega / \Omega_{\text {crit }}$, the third and fourth the maximum central temperature (in units of $10^{9} \mathrm{~K}$ ) and density (in units of $10^{5} \mathrm{~g} \mathrm{~cm}^{-3}$ ) that were encountered due to the pair-formation dynamical instability, the fifth the mass of the oxygen core, $M_{\mathrm{O} \text {-core (in units of }}$ solar masses), that each model produced, the sixth the surface abundance of ${ }^{14} \mathrm{~N}$, and the seventh the final fate of the model as observed in the FLASH hydrodynamics simulations ( $\mathrm{CC}$ for core collapse, PPISN for pulsational pair-instability supernova, and PISN for pair-instability supernova). In all figures, the models that ended as CC will be represented by black curves, the models that encountered PPISN by green curves, and the models that underwent PISN with red curves.

To compare with previous results for PPISN presented by Woosley et al. (2007) in the case of a $110 M_{\odot}$ ZAMS star and for direct PISN presented by Heger \& Woosley (2002) for a variety of stars with $M_{\text {ZAMS }}>140 M_{\odot}$ we run our own nonrotating $110 M_{\odot}$ and $200 M_{\odot}$ models. The model with $110 M_{\odot}$ formed a $56 M_{\odot}$ oxygen core and then encountered a violent pulsation that heated the center of the star up to $2.46 \times 10^{9} \mathrm{~K}$, in a similar manner to that suggested by Woosley et al. (2007). The $200 M_{\odot}$ model produced a direct PISN that synthesized a massive amount of ${ }^{56} \mathrm{Ni}\left(\sim 21 M_{\odot}\right)$ and totally disrupted the star, behaving exactly as predicted by Woosley et al. (2007). Additionally, in order to establish a lower mass end for the production of PPISNe, we have run a $35 M_{\odot}$ model with rotation at $80 \%$ of the critical velocity that converted essentially all of its mass to oxygen. This model was able to evolve up to $\mathrm{CC}$ in MESA. For benchmarking it was also mapped to FLASH at the time of core oxygen ignition where it also kept slowly evolving toward higher densities and temperatures always avoiding the pair-formation regime.

For all the models, increased levels of rotation led to a more chemically homogeneous evolution and produced higher oxygen core masses. ZAMS rotation at $80 \%$ of the critical Keplerian velocity was able to convert all stellar mass into oxygen for all the models. The evolution of some models rotating at $80 \%$ yielded brief stages when rotation became mildly super-critical (110\%-120\%) at the surface, which means that results from these models may not be completely accurate. For all other degrees of rotation the star remained at subcritical velocities throughout all of its evolutionary track. As a representative example of this effect, we show the evolution of $\Omega / \Omega_{\text {crit }}$ for the rotating $70 M_{\odot}$ models during the MS in Figure 1. The solid green curve, the dashed green curve, and the solid red curve show the evolution of $\Omega / \Omega_{\text {crit }}$ for ZAMS rotation at $30 \%, 50 \%$, and $80 \%$, respectively.

In order to illustrate the effects of rotation on a ZAMS star with a specific mass, we pick the $70 M_{\odot}$ models because, as can be seen in Table 1, all possible final fates are encountered for this model (CC for zero rotation, PPISN for $30 \%$ and $50 \%$ critical rotation, and PISN for $80 \%$ critical rotation). The evolution of the central density $\left(\rho_{c}\right)$ and temperature $\left(T_{c}\right)$ for these models is presented in the left panel of Figure 2. As can be seen, all rotating models encounter a collapse that heats the core up to higher densities and temperatures, but the one rotating at $80 \%$ critical is heated significantly enough to burn oxygen explosively and subsequently ejects of all its mass. Higher degrees of rotation lead the $\rho_{c}-T_{c}$ track of the star closer to the $\Gamma_{1}<4 / 3$ pairformation region. The right panel of Figure 2 shows the tracks of the models in the Hertzsprung-Russell diagram. It can be seen that the more rapidly rotating models remain bluer and 
Table 1

Characteristics of the Stellar Models Discussed in This Work

\begin{tabular}{|c|c|c|c|c|c|c|}
\hline $\begin{array}{l}M_{\text {ZAMS }} \\
\left(M_{\odot}\right) \\
\end{array}$ & $\Omega / \Omega_{\text {crit,ZAMS }}$ & $T_{9, \max }{ }^{\mathrm{a}}$ & $\rho_{5, \max } \mathrm{b}$ & $\begin{array}{c}M_{\mathrm{O} \text {-core }} \\
\left(M_{\odot}\right) \\
\end{array}$ & $\begin{array}{c}X_{14} N, \text { surf } \\
\left(10^{-3}\right)\end{array}$ & Fate $^{\mathrm{d}}$ \\
\hline 35 & 0.8 & $\ldots$ & $\ldots$ & 35 & 4.70 & $\mathrm{CC}$ \\
\hline 40 & 0.0 & $\ldots$ & $\ldots$ & 14 & 0.00 & $\mathrm{CC}$ \\
\hline 40 & 0.3 & $\ldots$ & $\ldots$ & 25 & 0.43 & $\mathrm{CC}$ \\
\hline 40 & 0.5 & $\ldots$ & $\ldots$ & 38 & 22.64 & $\mathrm{CC}$ \\
\hline 40 & 0.8 & 1.16 & 1.03 & 40 & 1.48 & PPISN \\
\hline 45 & 0.0 & $\ldots$ & $\ldots$ & 17 & 0.00 & $\mathrm{CC}$ \\
\hline 45 & 0.3 & $\ldots$ & $\ldots$ & 30 & 1.31 & $\mathrm{CC}$ \\
\hline 45 & 0.5 & 1.21 & 0.63 & 44 & 15.54 & PPISN \\
\hline 45 & 0.8 & 0.90 & 0.27 & 45 & 5.49 & PPISN \\
\hline 50 & 0.0 & $\ldots$ & $\ldots$ & 19 & 0.00 & $\mathrm{CC}$ \\
\hline 50 & 0.3 & $\ldots$ & $\ldots$ & 39 & 0.18 & $\mathrm{CC}$ \\
\hline 50 & 0.5 & 3.19 & 9.25 & 48 & 20.01 & PPISN \\
\hline 50 & 0.8 & 1.17 & 1.85 & 50 & 1.32 & PPISN \\
\hline 55 & 0.0 & $\ldots$ & $\ldots$ & 22 & 0.00 & $\mathrm{CC}$ \\
\hline 55 & 0.3 & 2.04 & 3.53 & 41 & 0.15 & PPISN \\
\hline 55 & 0.5 & 3.22 & 9.57 & 53 & 14.08 & PPISN \\
\hline 55 & 0.8 & 1.08 & 0.88 & 55 & 1.66 & PPISN \\
\hline 60 & 0.0 & $\ldots$ & $\ldots$ & 30 & 0.00 & $\mathrm{CC}$ \\
\hline 60 & 0.3 & 2.09 & 3.31 & 45 & 0.04 & PPISN \\
\hline 60 & 0.5 & 2.91 & 8.09 & 57 & 26.90 & PPISN \\
\hline 60 & 0.8 & 1.05 & 0.53 & 60 & 15.28 & PPISN \\
\hline 65 & 0.0 & $\ldots$ & $\ldots$ & 32 & 0.00 & $\mathrm{CC}$ \\
\hline 65 & 0.3 & 2.37 & 5.37 & 46 & 0.00 & PPISN \\
\hline 65 & 0.5 & 3.49 & 12.16 & 60 & 26.51 & PPISN \\
\hline 65 & 0.8 & 4.45 & 35.6 & 65 & 0.00 & PISN \\
\hline 70 & 0.0 & $\ldots$ & $\ldots$ & 35 & 0.00 & $\mathrm{CC}$ \\
\hline $70^{\mathrm{e}}$ & 0.0 & $\ldots$ & $\ldots$ & 30 & 0.00 & $\mathrm{CC}$ \\
\hline 70 & 0.3 & 2.58 & 6.90 & 48 & 0.00 & PPISN \\
\hline 70 & 0.5 & 3.03 & 9.22 & 66 & 20.58 & PPISN \\
\hline $70^{\mathrm{e}}$ & 0.5 & 3.02 & 9.20 & 65 & 18.12 & PPISN \\
\hline $70^{f}$ & 0.5 & $\ldots$ & $\ldots$ & 16 & 10.51 & $\mathrm{CC}$ \\
\hline 70 & 0.8 & 5.08 & 43.18 & 70 & 5.30 & PISN \\
\hline 75 & 0.0 & $\ldots$ & $\ldots$ & 36 & 0.00 & $\mathrm{CC}$ \\
\hline 75 & 0.3 & 3.21 & 10.40 & 54 & 0.02 & PPISN \\
\hline 75 & 0.5 & 3.61 & 20.00 & 67 & 16.68 & PISN \\
\hline 75 & 0.8 & 5.28 & 61.16 & 75 & 0.00 & PISN \\
\hline 80 & 0.0 & 1.87 & 2.44 & 40 & 0.00 & PPISN \\
\hline 80 & 0.3 & 1.99 & 2.30 & 59 & 0.00 & PPISN \\
\hline 80 & 0.5 & 4.31 & 20.36 & 77 & 26.41 & PISN \\
\hline 80 & 0.8 & 2.97 & 8.99 & 80 & 0.00 & PISN \\
\hline 85 & 0.0 & 2.09 & 3.61 & 40 & 0.00 & PPISN \\
\hline 85 & 0.3 & 1.53 & 0.81 & 65 & 0.39 & PISN \\
\hline 85 & 0.5 & 3.46 & 10.41 & 79 & 24.93 & PISN \\
\hline 85 & 0.8 & 3.25 & 9.84 & 85 & 8.64 & PISN \\
\hline 90 & 0.0 & 1.86 & 1.76 & 45 & 0.00 & PPISN \\
\hline 90 & 0.3 & 3.98 & 17.44 & 85 & 14.24 & PISN \\
\hline 95 & 0.0 & 2.75 & 6.59 & 50 & 0.00 & PPISN \\
\hline 95 & 0.3 & 4.62 & 26.97 & 90 & 33.25 & PISN \\
\hline 110 & 0.0 & 2.46 & 5.85 & 56 & 0.00 & PPISN \\
\hline 200 & 0.0 & 5.02 & 34.40 & 120 & 0.00 & PISN \\
\hline
\end{tabular}

Notes.

${ }^{\text {a }}$ In units of $10^{9} \mathrm{~K}$.

${ }^{\mathrm{b}}$ In units of $10^{5} \mathrm{~g} \mathrm{~cm}^{-3}$.

${ }^{\mathrm{c}}$ We adopt mass fraction of 0.00 to be anything less than $10^{-6}$.

${ }^{\mathrm{d}} \mathrm{CC}$ : core collapse; PPISN: pulsational pair-instability supernova; PISN: pair-instability supernova.

${ }^{\mathrm{e}}$ With Ledoux criterion for mixing implemented.

${ }^{\mathrm{f}}$ For $Z=Z_{\odot}$ and mass loss. 


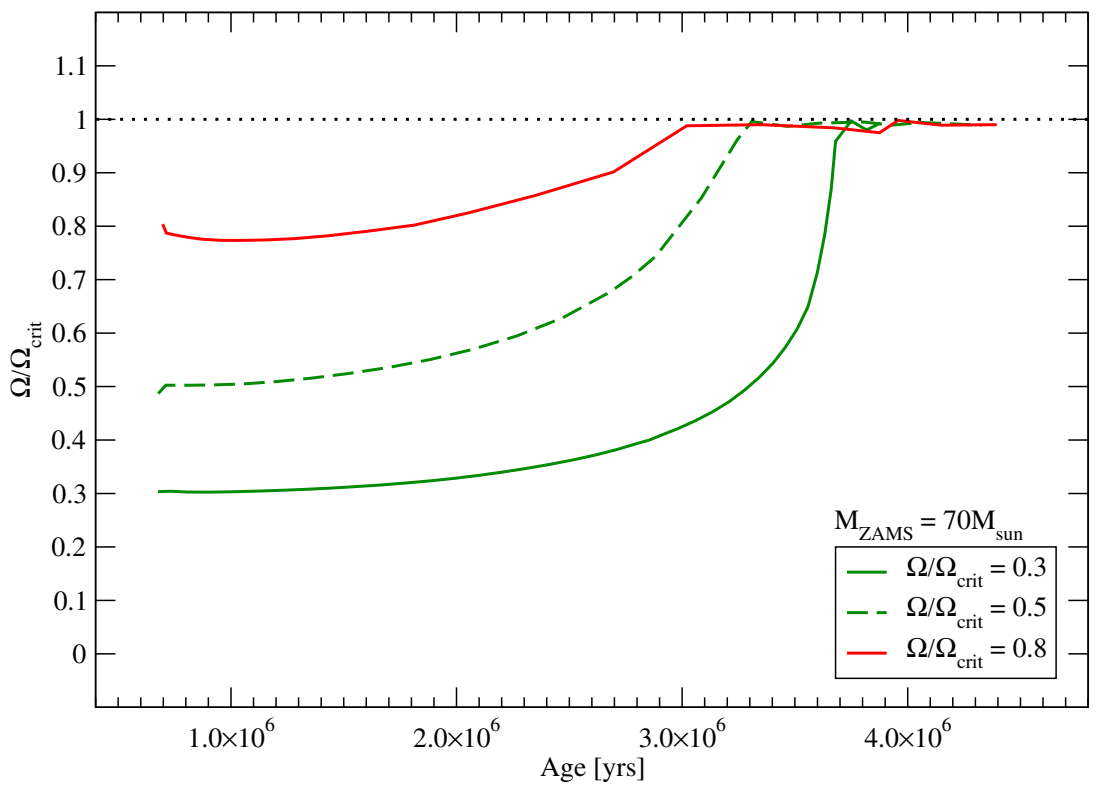

Figure 1. Evolution of $\Omega / \Omega_{\text {crit }}$ at the surface for the $70 M_{\odot}$ models for initial ZAMS $\Omega / \Omega_{\text {crit }}=0.3$ (solid green curve), $\Omega / \Omega_{\text {crit }}=0.5$ (dashed green curve), and $\Omega / \Omega_{\text {crit }}=0.8$ (solid red curve).

(A color version of this figure is available in the online journal.)
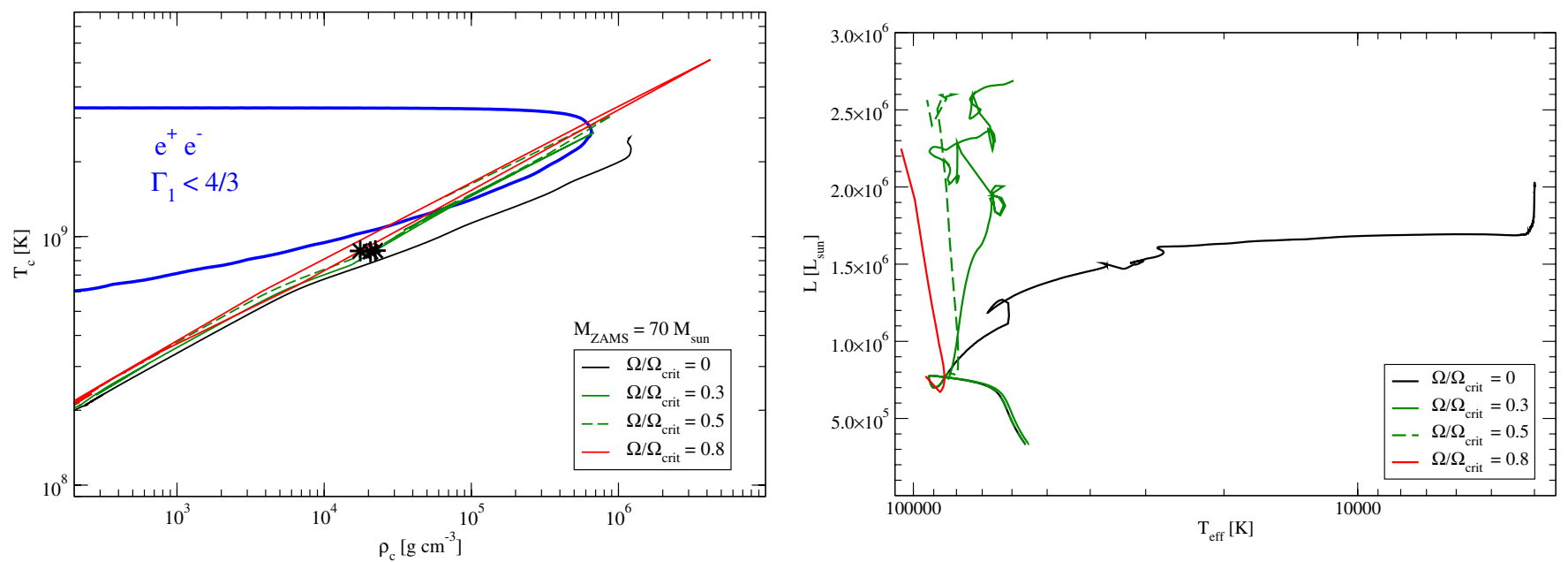

Figure 2. Left panel: central density and temperature evolution of the $70 M_{\odot}$ (Schwarzschild) models for $\Omega / \Omega_{\text {crit }}=0$ (solid black curve), $\Omega / \Omega_{\text {crit }}=0.3$ (solid green curve), $\Omega / \Omega_{\text {crit }}=0.5$ (dashed green curve), and $\Omega / \Omega_{\text {crit }}=0.8$ (solid red curve). The solid blue curve marks the electron-positron pair-instability region where the adiabatic index is $\Gamma_{1}<4 / 3$. The black stars mark the point where the models were mapped to the hydrodynamics code. Right panel: evolution of the $70 M_{\odot}$ (Schwarzschild) models in the H-R diagram.

(A color version of this figure is available in the online journal.)

are more luminous than the less rapidly rotating ones. Figure 3 illustrates the chemical composition of the $70 M_{\odot}$ models for all degrees of rotation at the time before core oxygen ignition (upper left panel: no rotation; upper right panel: $30 \%$ critical rotation; lower left panel: $50 \%$ critical rotation; lower right panel: $80 \%$ critical rotation). The effects of increased homogeneity and higher $M_{\mathrm{O}-\text { core }}$, with increasing rotation are clearly illustrated. Figure 4 presents the distribution of the $\mathrm{CNO}$ (solid black curve) and 3- $\alpha$ process specific nuclear energy inputs are shown.

Higher degrees of rotation also produced a significantly higher ${ }^{14} \mathrm{~N}$ surface abundance than their non-rotating counterparts, a trend also noted by Ekström et al. (2008). This trend, however, is not monotonic. For the extreme level of $80 \%$ critical rotation the surface ${ }^{14} \mathrm{~N}$ mass fraction is generally found to be reduced while the most significant ${ }^{14} \mathrm{~N}$ enrichment is observed in models rotating at $50 \%$ of the critical value. Increased surface
${ }^{14} \mathrm{~N}$ is attributed to the onset of the $\mathrm{CNO}$ cycle in the outer shell due to the strong rotationally induced mixing. For completely homogeneous evolution that makes an oxygen star, which takes place for models that rotate at the extreme level of $80 \%$ critical rotation, the $\mathrm{CNO}$ contribution in outer layers of the star is confined to a very thin shell.

Figure 5 illustrates our final results for the fate of rotating massive primordial stars. As can be seen, stars initially rotating with speeds $80 \%$ the critical velocity with $40 M_{\odot}<M_{\text {ZAMS }}<$ $60 M_{\odot}$ will produce PPISNe associated with episodic mass loss and for $M_{\text {ZAMS }} \geqslant 65 M_{\odot}$ they will explode as PISNe. As mentioned before, at this rapid rotation the whole mass of those stars will turn into oxygen, therefore this result is consistent with the findings of Heger et al. (2003), but for initial ZAMS masses that are only $40 \%-50 \%$ those of the ones given by their nonrotating calculations. Consequently, for this "fiducial" degree of 

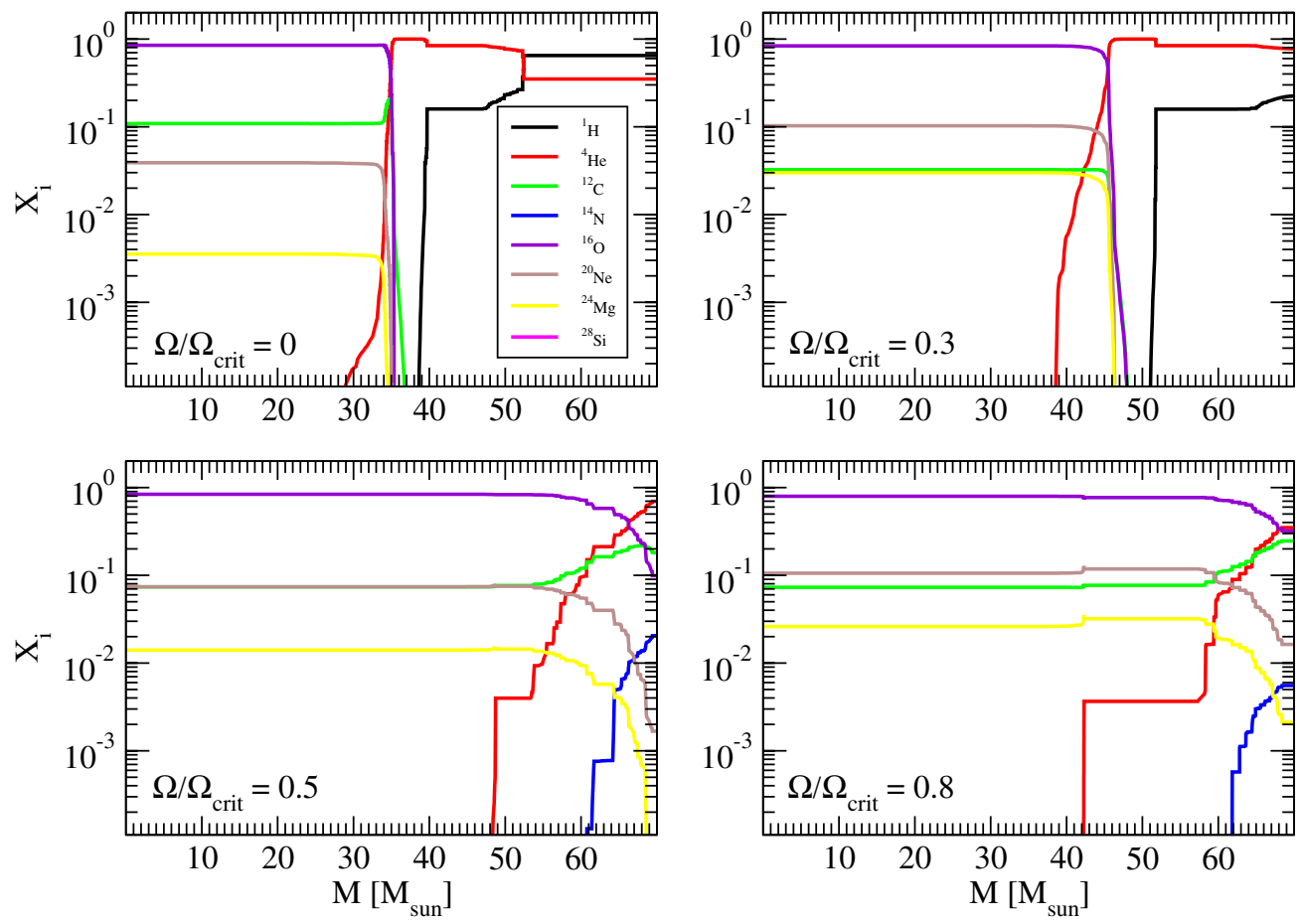

Figure 3. Chemical composition of the $70 M_{\odot}$ (Schwarzschild) models for $\Omega / \Omega_{\text {crit }}=0$ (upper left panel), $\Omega / \Omega_{\text {crit }}=0.3$ (upper right panel), $\Omega / \Omega_{\text {crit }}=0.5$ (lower left panel), and $\Omega / \Omega_{\text {crit }}=0.8$ (lower right panel) at the time just prior to core oxygen ignition. The specific elements plotted are given in the inset in the upper left panel. (A color version of this figure is available in the online journal.)
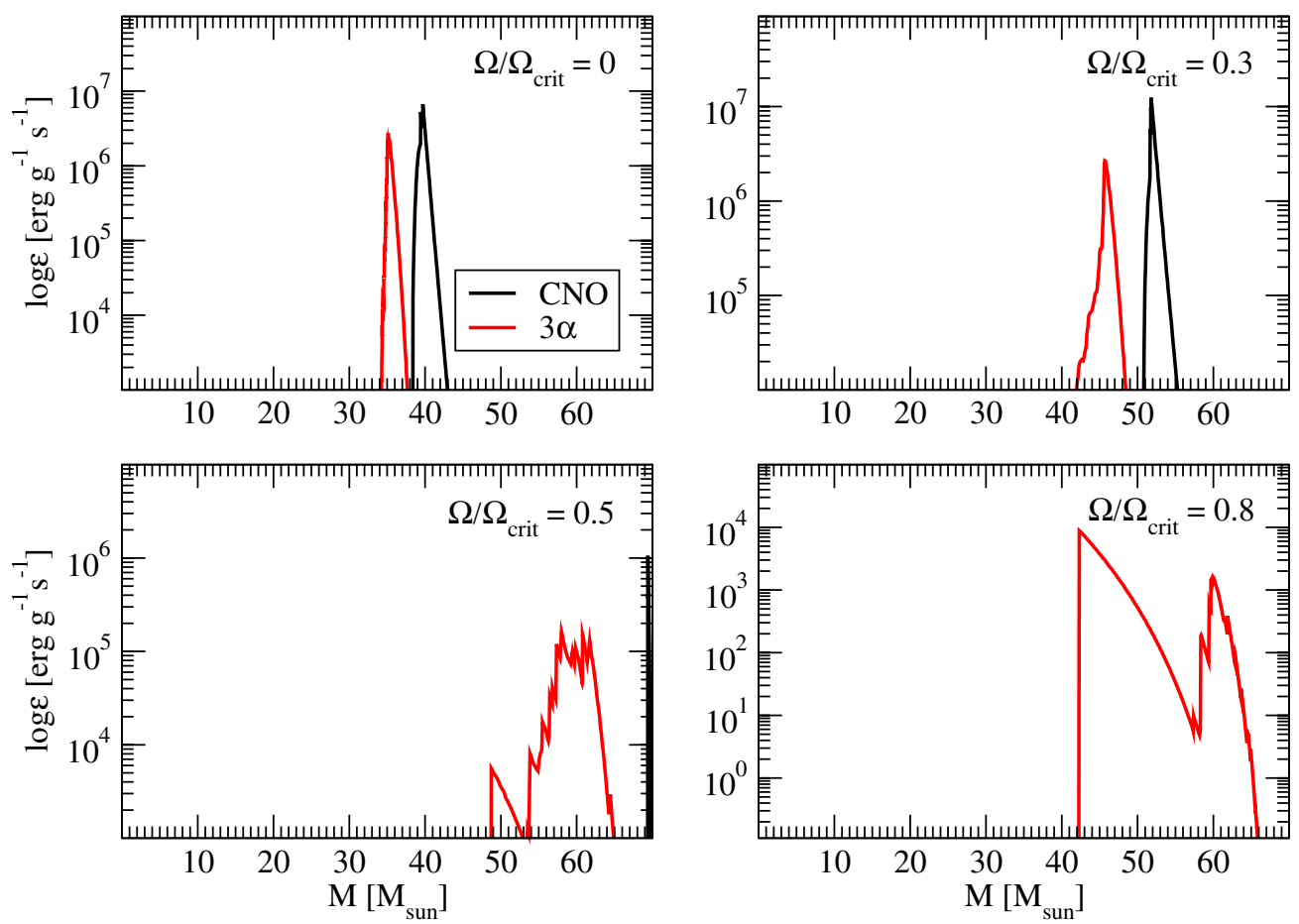

Figure 4. Specific nuclear energy generation input mass distributions for the $70 M_{\odot}$ (Schwarzschild) models (CNO cycle: solid black curve; triple-alpha process: solid red curve) for $\Omega / \Omega_{\text {crit }}=0$ (upper left panel), $\Omega / \Omega_{\text {crit }}=0.3$ (upper right panel), $\Omega / \Omega_{\text {crit }}=0.5$ (lower left panel), and $\Omega / \Omega_{\text {crit }}=0.8$ (lower right panel) at the time just prior to core oxygen ignition.

(A color version of this figure is available in the online journal.)

rotation at $50 \%$ the critical value, the ZAMS mass limits become $45 M_{\odot}<M_{\text {ZAMS }}<70 M_{\odot}$ for PPISN and $M_{\text {ZAMS }} \geqslant 75 M_{\odot}$ for PISN progenitors, thus at the level of 50\%-60\% of those in the case of no rotation. These results suggest that episodic mass-loss events resulting from PPISNe can be encountered for less massive stars and may account for some of the observed luminous blue variable (LBV) type events. We wish to add that a recent paper by Yoon et al. (2012) independently calculated the mass limits of PPISN and PISN primordial progenitors and found good agreement with our results. 


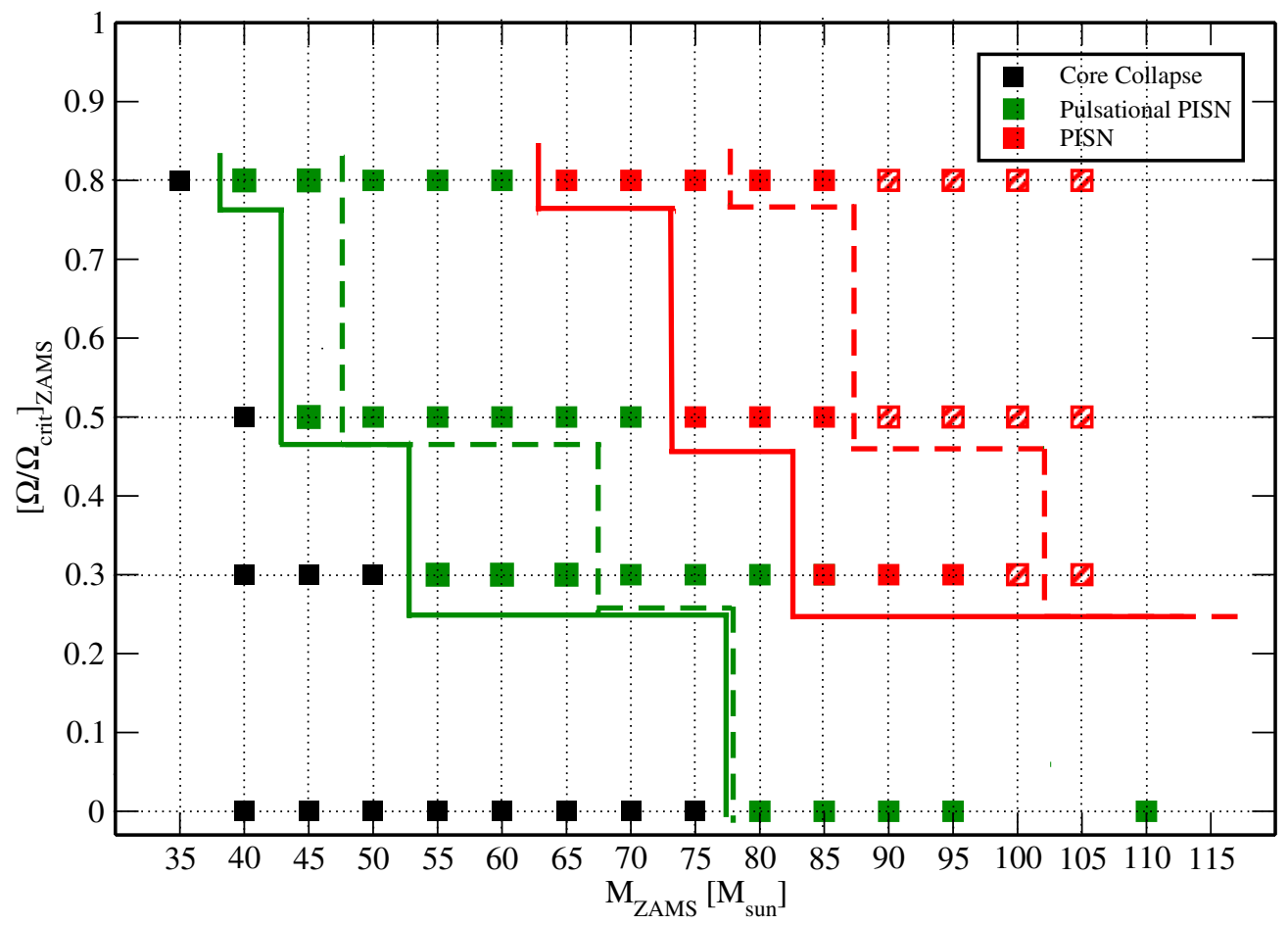

Figure 5. Final fate of high ZAMS mass stellar models for different degrees of ZAMS rotation. Filled black squares indicate core collapse (CC), filled green squares indicate pulsational pair-instability supernova (PPISN), and filled red squares direct pair-instability supernova (PISN) explosion. The hatched red squares indicate the PISN fate of the $Z=0$ models run only with mass loss included in the calculations. The dotted lines mark the ZAMS mass and rotation level grid for the models done in this work. The thick green and red lines mark the approximate boundaries between the different final fates of the models. The dashed thick green and red lines mark those same approximate boundaries in the case where mass loss at $Z=0$ is considered in the calculations.

(A color version of this figure is available in the online journal.)

\subsection{Effects of Mixing}

Convective mixing and overshoot are some of the factors that control the final mass of the oxygen core. As mentioned above, in this work we have adopted the Schwarzschild criterion for convection based on the lack of true knowledge about the nature of convection in realistic three-dimensional situations. 1D convective mixing will be suppressed if composition gradients are considered, as suggested by the Ledoux criterion for convection. This will result in smaller final oxygen core masses and therefore tracks in the $\rho_{c}-T_{c}$ plane that are shifted further away from the pair-formation $\Gamma_{1}<4 / 3$ region, something that may alter the results for the minimum ZAMS masses that encounter PPISN and PISN.

We ran MESA for the $70 M_{\odot}$ non-rotating and $50 \%$ critically rotating models with the Ledoux criterion implemented in order to examine the sign of this effect. Figure 6 presents the results in the case of zero rotation. The left panel shows the $\rho_{c}-T_{c}$ tracks for the model that uses the Schwarzschild (solid black curve) and the model that uses the Ledoux (dashed black curve) criterion. The right upper and lower panels show the chemical composition of the two models at the time before core oxygen ignition. It can be seen that when mixing is suppressed the nonrotating $70 M_{\odot}$ model evolves toward slightly lower $\rho_{c}$ and $T_{c}$ values and it ends up making an oxygen core that is $86 \%$ the one produced in the case where composition gradients are ignored. The result of the same experiment but in the case of rotation at the $50 \%$ of the critical value is shown in Figure 7. In this case, we see that the fast rotationally induced mixing counters the effects of suppression due to the inclusion of composition gradients and the final oxygen core masses are almost equal. The characteristics of the models run with the Ledoux criterion implemented are also given in Table 1. These results indicate that selecting a different $1 \mathrm{D}$ prescription for convective mixing is unlikely to alter our results for the rotating models by any significant factor.

\subsection{Effects of Metallicity and Mass Loss}

Although a thorough study of massive models with $Z>0$ is beyond the scope of this project, which focuses on the primordial progenitors of PISNe, we considered the effects that the presence of metals may have. As is well known, metallicities with $Z>0$ can induce significant line-driven mass loss that can cause massive stars to lose a significant amount of mass. This mass loss will drive evolution toward the formation of oxygen cores with smaller mass than in the case of zero mass loss. This will have an effect on the final fate of a very massive star, such that it might miss the pair-formation region in the $\rho_{c}-T_{c}$ plane and end its life as a CC SN explosion. The high-mass loss may lead to the formation of massive circumstellar material (CSM) environments around the progenitors of these SN so that the SN ejecta will violently interact with the CSM producing shock energy that can power their LC, as manifested by luminous Type IIn SNe. Evolution of massive PISN progenitors at higher metallicities has been studied in more detail by Langer et al. (2007) where a metallicity threshold of $Z \sim Z_{\odot} / 3$ is determined below which PISN may occur.

To account for the mass loss with MESA, the prescriptions of de Jager et al. (1988) were used, as appropriate for hot O-type stars (for a recent discussion on mass-loss rates for Wolf-Rayet stars, see Yoon et al. 2010). Figure 5 also shows the final fate of the same rotating massive zero-metallicity stars for which mass loss is considered in the calculations. As mentioned above, 

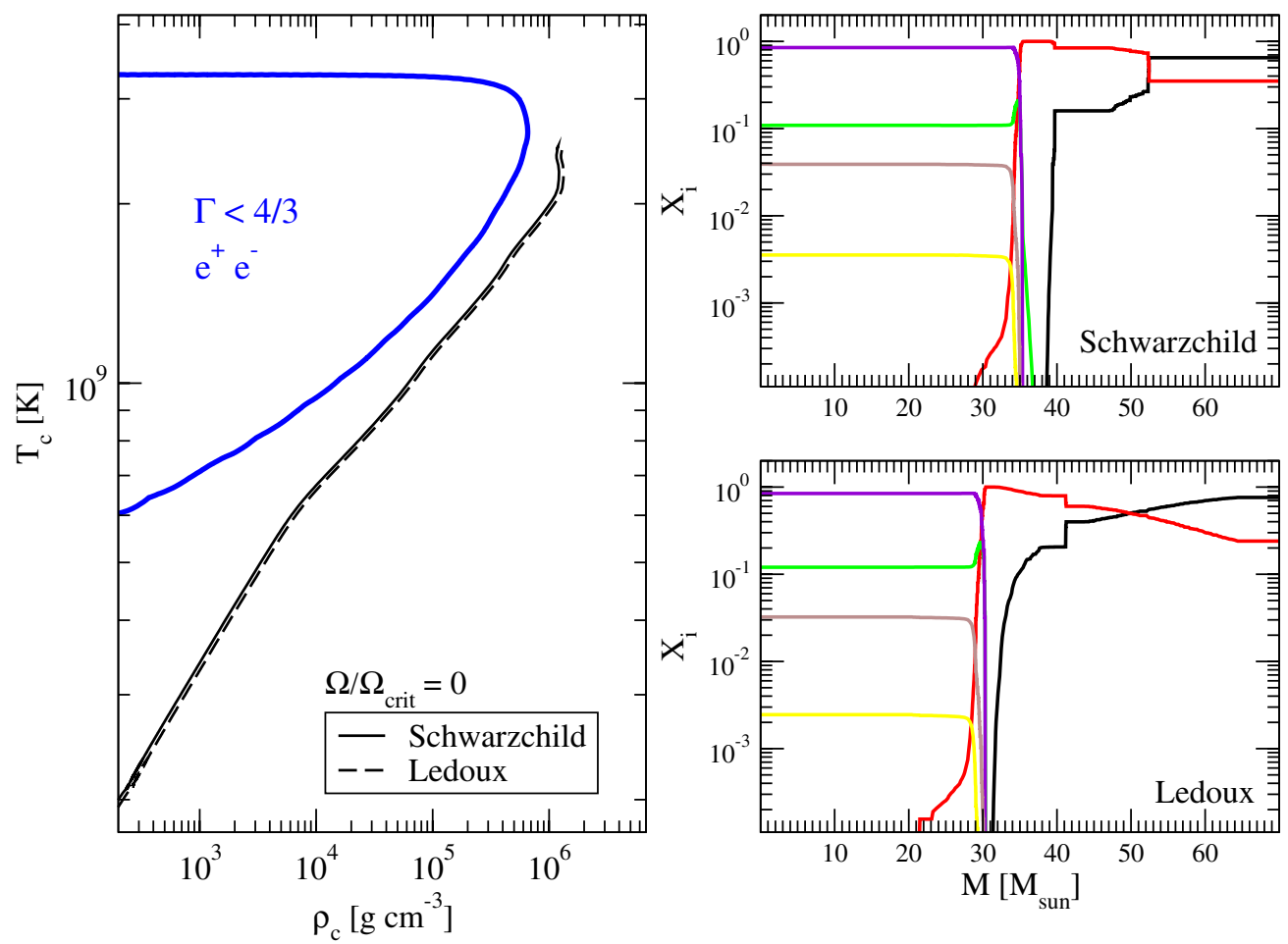

Figure 6. Left panel: central density and temperature evolution of the $70 M_{\odot}, \Omega / \Omega_{\text {crit }}=0$ models implementing the Schwarzschild (solid black curve) and Ledoux (dashed black curve) mixing criteria. The solid blue curve marks the electron-positron pair-instability region where the adiabatic index is $\Gamma_{1}<4 / 3$. Upper right panel: chemical composition of the $70 M_{\odot}, \Omega / \Omega_{\text {crit }}=0$ model with the Schwarzschild mixing criterion implemented at the time just prior to core oxygen ignition. Lower right panel: chemical composition of the $70 M_{\odot}, \Omega / \Omega_{\text {crit }}=0$ model with the Ledoux mixing criterion implemented at the time just prior to core oxygen ignition. The specific elements plotted are given in the inset in the upper left panel of Figure 3.

(A color version of this figure is available in the online journal.)
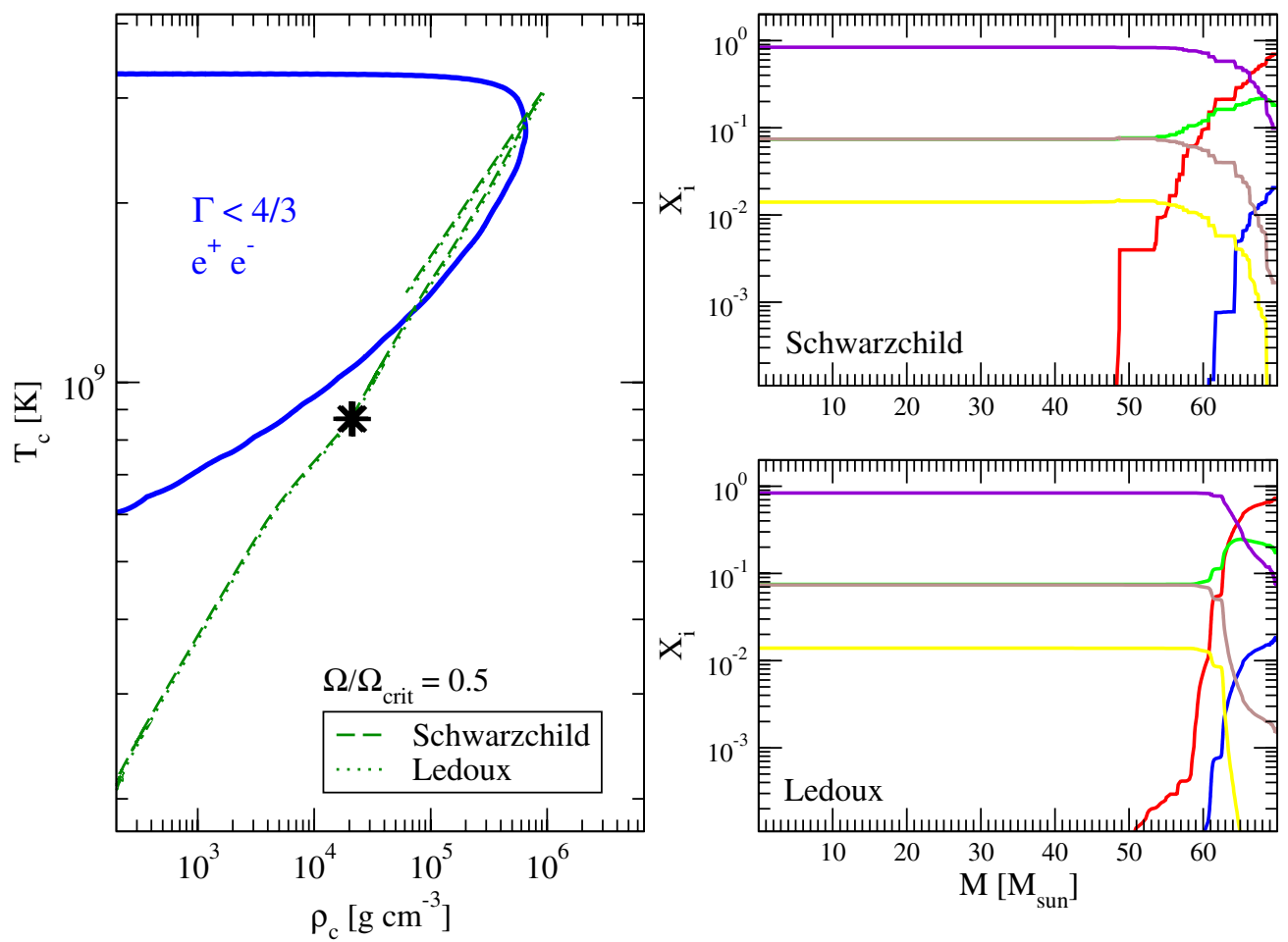

Figure 7. Left panel: central density and temperature evolution of the $70 M_{\odot}, \Omega / \Omega_{\text {crit }}=0.5$ models implementing the Schwarzschild (dashed green curve) and Ledoux (dotted green curve) mixing criteria. The solid blue curve marks the electron-positron pair-instability region where the adiabatic index is $\Gamma_{1}<4 / 3$. The black stars mark the point where the models were mapped to the hydrodynamics code. Upper right panel: chemical composition of the $70 M_{\odot}, \Omega / \Omega_{\text {crit }}=0.5$ model with the Schwarzschild mixing criterion implemented at the time just prior to core oxygen ignition. Lower right panel: chemical composition of the $70 M_{\odot}, \Omega / \Omega_{\text {crit }}=0.5$ model with the Ledoux mixing criterion implemented at the time just prior to core oxygen ignition. The specific elements plotted are given in the inset in the upper left panel of Figure 3.

(A color version of this figure is available in the online journal.) 

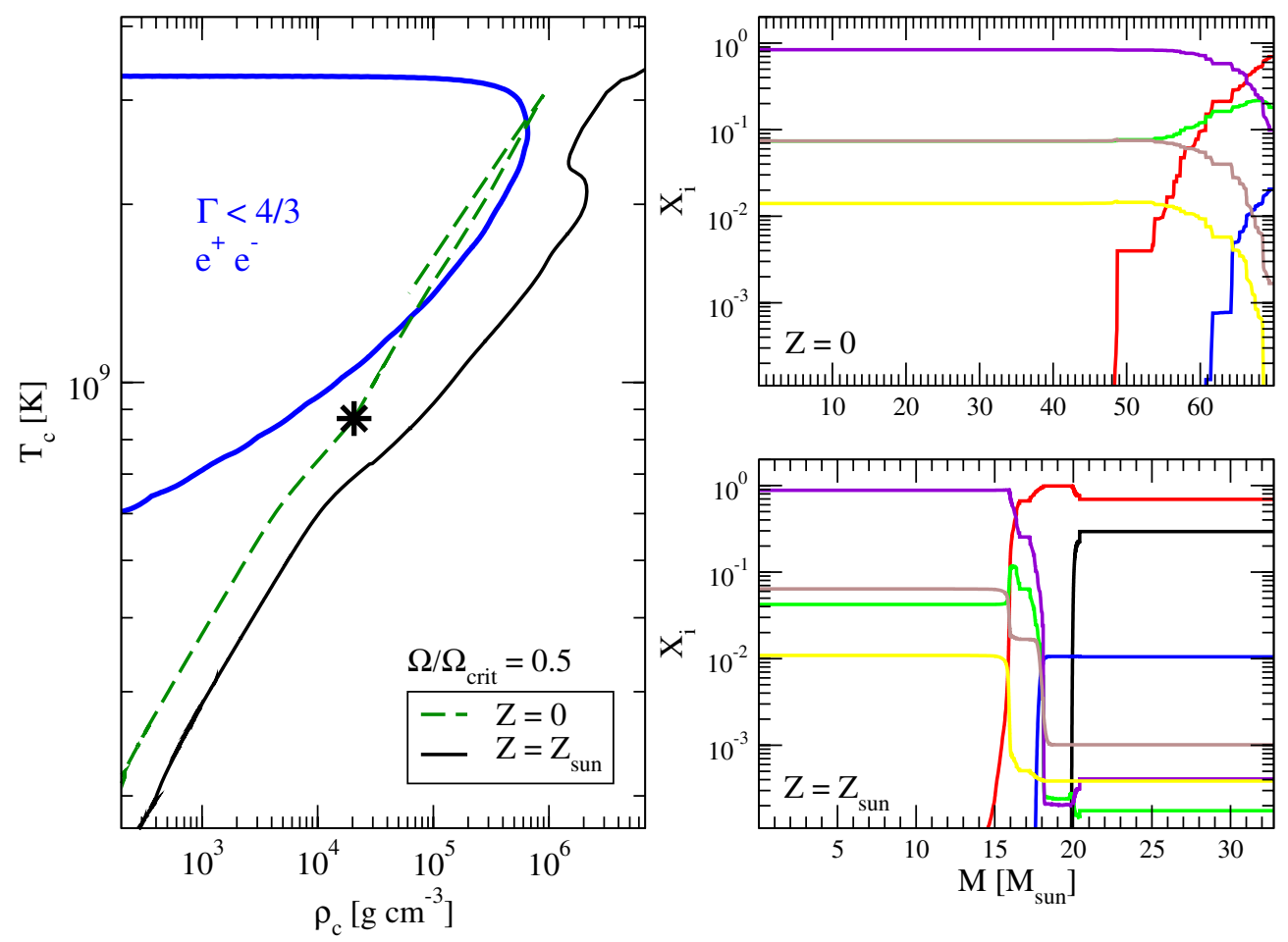

Figure 8. Left panel: central density and temperature evolution of the $70 M_{\odot}, \Omega / \Omega_{\text {crit }}=0.5$ models for $Z=0$ (dashed green curve) and $Z=Z Z_{\odot}$ (solid black curve) using the Schwarzschild criterion and the de Jager et al. (1988) mass-loss prescriptions. The solid blue curve marks the electron-positron pair-instability region where the adiabatic index is $\Gamma_{1}<4 / 3$. The black stars mark the point where the models were mapped to the hydrodynamics code. Upper right panel: chemical composition of the $70 M_{\odot}$ for $Z=0$ at the time before core oxygen ignition. Lower right panel: chemical composition of the $70 M_{\odot}$ for $Z=Z_{\odot}$ at the time before core oxygen ignition. The specific elements plotted are given in the inset in the upper left panel of Figure 3.

(A color version of this figure is available in the online journal.)

rotationally induced mixing will dredge up metals to the outer and less gravitationally bound regions of the star inducing linedriven mass loss that will ultimately result in smaller oxygen core mass. We find that when mass loss is considered, the minimum ZAMS mass that produce PPISN events is $\sim 50 M_{\odot}$ and the minimum mass that produce PISN events is $\sim 80 M_{\odot}$ at maximum ZAMS rotation ( $80 \%$ of the critical value). The results for the fate of the $70 M_{\odot}$ model rotating at $50 \%$ the critical value with solar metallicity and mass loss are presented in Figure 8. The left panel of Figure 8 shows a comparison between the $\rho_{c}-T_{c}$ tracks of the two models with (solid black curve) and without (solid green curve) mass loss, and the upper and lower right panels show the chemical composition of the models at the time before core oxygen ignition. The strong linedriven mass loss ejected the hydrogen and a fraction of the helium shell leaving a $33 M_{\odot}$ star with $M_{\mathrm{O} \text {-core }}=16 M_{\odot}$ that ended its life as a CC SN. The strong continuous mass loss led the $\rho_{c}-T_{c}$ track of the model further from the electron-positron pair $\Gamma_{1}<4 / 3$ region. Furthermore, due to strong rotationally induced mixing, the pre-SN model had an enhanced ${ }^{14} \mathrm{~N}$ surface abundance as was the case with most rotating $Z=0$ models. The extreme amount of mass lost by the star that we compute here is consistent with results presented by Ekström et al. (2011) who calculated the evolution of a grid of solar metallicity models from 0.8 to $120 M_{\odot}$ rotating at $40 \%$ the critical velocity. Ekström et al. (2011) found that even the most massive rotating $120 M_{\odot}$ model ends its life with a mass of $19 M_{\odot}$, way below the limit for PPISN. This result indicates that PPISN and, even more so, PISN events that may result from local $\left(Z \sim Z_{\odot}\right)$ massive progenitors must be very rare compared to those that result from metal-poor primordial progenitors.

\section{DISCUSSION AND CONCLUSIONS}

Recently, Stacy et al. (2011) determined the rotational speed of massive primordial stars to be close to $50 \%$ of the critical value using multi-dimensional smooth particle hydrodynamics (SPH) simulations. In addition, observational evidence presented by Dufton et al. (2011) on the 20-30 $M_{\odot}$ late O-type star VFTS102 in 30 Doradus indicates a rotational speed $>50 \%$ of the critical value for this star. VFTS102 has $Z>0$ and is not massive enough to encounter pair formation in any circumstances, but it establishes the existence of fast rotation in some high-mass stars. Using MESA to follow the evolution and FLASH to compute the late-time hydrodynamics for a grid of massive primordial $(Z=0)$ stars with various degrees of rotation and including the effects of magnetic fields as currently parameterized, we established the ZAMS mass ranges required to produce PPISN or PISN as presented in Figure 5. For significant rotational velocities, we find that stars with ZAMS masses as low as $40-45 M_{\odot}$ can produce PPISN and stars with ZAMS masses $>65-75 M_{\odot}$ can produce direct PISN explosions due to a more chemically homogeneous evolution that leads to increased oxygen core masses.

We also investigated the effects of convective mixing, metallicity, and mass loss. We found that suppressed mixing due to composition gradients is unlikely to significantly alter the results for the rotating models since rotationally induced mixing is the dominant factor. We also found that higher metallicities (and specifically solar metallicity) can induce extreme linedriven mass loss so that even some of the most massive ZAMS stars end their lives as $\mathrm{CC} \mathrm{SNe}$ avoiding the pair-formation 
instability. Current results from numerical simulations (Greif et al. 2011) suggest a nearly flat primordial initial mass function (IMF) with typical mass of $100 M_{\odot}$. If so, PISN and especially PPISN events may be more frequent than estimates suggest based on non-rotating models (Scannapieco et al. 2005). This raises the possibility of detecting a larger number of those spectacular explosions with future missions such the James Webb Space Telescope (JWST).

As emphasized by Smith \& Owocki (2006) and Smith et al. (2007), stars under a wide variety of conditions may undergo the mass ejection process associated with LBVs. The physical mechanism of the LBV phenomenon is not well understood. PPISN is not likely to be the only mechanism involved in the LBV process, but our result that stars with initial mass as low as $40 M_{\odot}$ may undergo PPISN means that PPISN should be considered as a possible candidate mechanism for the LBV mass-loss phenomenon in some circumstances. It would be worthwhile to explore the PPISN process in stars with a variety of envelope compositions and structures, including those that are nearly pure, bare, oxygen cores to understand how PPISN may lead to single or multiple shell-ejection phases.

We thank the MESA team for making this valuable tool readily available and we especially thank Bill Paxton for his ready advice and council in running the code. We wish to thank the anonymous referee for useful suggestions and comments. We also wish to thank Volker Bromm and Athena Stacy for discussions of the formation and evolution of Pop III stars. E.C. thanks Tuguldur Sukhbold for help with MESA and Selma de Mink for useful suggestions. E.C. also thanks Sean Couch and Christopher Lindner for help with FLASH. J.C.W. especially thanks Zalman Barkat for first introducing him to this topic and for sharing portions of his PhD thesis. This research is supported in part by NSF AST-1109801.

\section{REFERENCES}

Abel, T., Anninos, P., Norman, M. L., \& Zhang, Y. 1998, ApJ, 508, 518 Abel, T., Bryan, G. L., \& Norman, M. L. 2000, ApJ, 540, 39 Appenzeller, I. 1970, A\&A, 5, 355

Barkat, Z., Rakavy, G., \& Sack, N. 1967, Phys. Rev. Lett., 18, 379

Bond, J. R., Arnett, W. D., \& Carr, B. J. 1984, ApJ, 280, 825

Bromm, V., Coppi, P. S., \& Larson, R. B. 2002, ApJ, 564, 23

Bromm, V., \& Larson, R. B. 2004, ARA\&A, 42, 79

Brott, I., de Mink, S. E., Cantiello, M., et al. 2011a, A\&A, 530, A115

Brott, I., Evans, C. J., Hunter, I., et al. 2011b, A\&A, 530, A116

Carr, B. J., Bond, J. R., \& Arnett, W. D. 1984, ApJ, 277, 445

Chatzopoulos, E., Wheeler, J. C., \& Vinko, J. 2012, arXiv:1111.5237

Chatzopoulos, E., Wheeler, J. C., Vinko, J., et al. 2011, ApJ, 729, 143

Chevalier, R. A., \& Irwin, C. M. 2011, ApJ, 729, L6

Christlieb, N., Bessell, M. S., Beers, T. C., et al. 2002, Nature, 419, 904
Crowther, P. A., Schnurr, O., Hirschi, R., et al. 2010, MNRAS, 408, 731

de Jager, C., Nieuwenhuijzen, H., \& van der Hucht, K. A. 1988, A\&AS, 72, 259

Dufton, P. L., Dunstall, P. R., Evans, C. J., et al. 2011, ApJ, 743, L22

Ekström, S., Georgy, C., Eggenberger, P., et al. 2011, arXiv:1110.5049

Ekström, S., Meynet, G., Chiappini, C., Hirschi, R., \& Maeder, A. 2008, A\&A, 489, 685

El Eid, M. F., Fricke, K. J., \& Ober, W. W. 1983, A\&A, 119, 54

El Eid, M. F., \& Hilf, E. R. 1977, A\&A, 57, 243

Fraley, G. S. 1968, Ap\&SS, 2, 96

Frebel, A., Aoki, W., Christlieb, N., et al. 2005, Nature, 434, 871

Fryxell, B., Olson, K., Ricker, P., et al. 2000, ApJS, 131, 273

Gal-Yam, A., Mazzali, P., Ofek, E. O., et al. 2009, Nature, 462, 624

Greif, T. H., Springel, V., White, S. D. M., et al. 2011, ApJ, 737, 75

Heger, A., Fryer, C. L., Woosley, S. E., Langer, N., \& Hartmann, D. H. 2003, ApJ, 591,288

Heger, A., Langer, N., \& Woosley, S. E. 2000, ApJ, 528, 368

Heger, A., \& Woosley, S. E. 2002, ApJ, 567, 532

Heger, A., \& Woosley, S. E. 2010, ApJ, 724, 341

Heger, A., Woosley, S. E., \& Spruit, H. C. 2005, ApJ, 626, 350

Hunter, D. A., Shaya, E. J., Holtzman, J. A., et al. 1995, ApJ, 448, 179

Joggerst, C. C., \& Whalen, D. J. 2011, ApJ, 728, 129

Kasen, D., Woosley, S. E., \& Heger, A. 2011, ApJ, 734, 102

Langer, N., Norman, C.-A., de Koter, A., et al. 2007, A\&AP, 475, L19

Leloudas, G., Chatzopoulous, E., Dilday, B., et al. 2012, A\&A, arXiv:1201.5393

Maeder, A. 1987, A\&A, 178, 159

Maeder, A., \& Meynet, G. 2011, arXiv:1109.6171

Miller, A. A., Chornock, R., Perley, D. A., et al. 2009, ApJ, 690, 1303

Ober, W. W., El Eid, M. F., \& Fricke, K. J. 1983, A\&A, 119, 61

Ohkubo, T., Nomoto, K., Umeda, H., Yoshida, N., \& Tsuruta, S. 2009, ApJ, 706,1184

Ohkubo, T., Umeda, H., \& Nomoto, K. 2003, Nucl. Phys. A, 718, 632

Panagia, N., Tanzi, E. G., \& Tarenghi, M. 1983, ApJ, 272, 123

Paxton, B., Bildsten, L., Dotter, A., et al. 2011, ApJS, 192, 3

Quimby, R. M., Aldering, G., Wheeler, J. C., et al. 2007, ApJ, 668, L99

Rakavy, G., \& Shaviv, G. 1967, ApJ, 148, 803

Rakavy, G., \& Shaviv, G. 1968, Ap\&SS, 1, 429

Rakavy, G., Shaviv, G., \& Zinamon, Z. 1967, ApJ, 150, 131

Scannapieco, E., Madau, P., Woosley, S., Heger, A., \& Ferrara, A. 2005, ApJ, 633, 1031

Smith, N., Li, W., Foley, R. J., et al. 2007, ApJ, 666, 1116

Smith, N., \& Owocki, S. P. 2006, ApJ, 645, L45

Spruit, H. C. 1999, A\&A, 349, 189

Spruit, H. C. 2002, A\&A, 381, 923

Stacy, A., Bromm, V., \& Loeb, A. 2011, MNRAS, 413, 543

Stacy, A., Greif, T. H., \& Bromm, V. 2010, MNRAS, 403, 45

Stringfellow, G. S., \& Woosley, S. E. 1988, in Origin and Distribution of the Elements, ed. G. J. Mathews (Singapore: World Scientific Publishing), 467 Timmes, F. X. 1999, ApJS, 124, 241

Timmes, F. X., \& Swesty, F. D. 2000, ApJS, 126, 501

Waldman, R. 2008, ApJ, 685, 1103

Whalen, D. J., \& Fryer, C. 2010, in AIP Conf. Proc. 1279, Deciphering the Ancient Universe with Gamma-Ray Bursts, ed. N. Kawai \& S. Nagataki (Melville, NY: AIP), 116

Wheeler, J. C. 1977, Ap\&SS, 50, 125

Woosley, S. E., Blinnikov, S., \& Heger, A. 2007, Nature, 450, 390

Yoon, S.-C., Dierks, A., \& Langer, N. 2012, arXiv:1201.2364

Yoon, S.-C., \& Langer, N. 2005, A\&A, 443, 643

Yoon, S.-C., Woosley, S. E., \& Langer, N. 2010, ApJ, 725, 940

Ziebarth, K. 1970, ApJ, 162, 947 